Article

\title{
Local Sharp Vector Variational Type Inequality and Optimization Problems
}

\author{
Jong Kyu Kim ${ }^{1, *}$ and Salahuddin ${ }^{2}$ (i) \\ 1 Department of Mathematics Education, Kyungnam University, Changwon, Gyeongnam 51767, Korea \\ 2 Department of Mathematics, Jazan University, Jazan-45142, Saudi Arabia; drsalah12@hotmail.com \\ * Correspondence: jongkyuk@kyungnam.ac.kr; Tel.: +82-10-4556-8608
}

Received: 4 September 2020; Accepted: 15 October 2020; Published: 20 October 2020

\begin{abstract}
In this paper, our goal was to establish the relationship between solutions of local sharp vector variational type inequality and sharp efficient solutions of vector optimization problems, also Minty local sharp vector variational type inequality and sharp efficient solutions of vector optimization problems, under generalized approximate $\eta$-convexity conditions for locally Lipschitzian functions.
\end{abstract}

Keywords: vector variational type inequality problems; vector optimization problems; efficient solutions; approximate $\eta$-convexity; Lipschitzian functions

\section{Introduction}

The research of variational inequality problems is a part of development in the theory of optimization since optimization problems can often be specialized to the solution of variational inequality problems. It is very important to point out that these theories pertain to more than just optimization problems and there in lies much of their attractiveness. Several authors have presented numerous fascinating results on variational inequality problems; see cited references here [1-12].

In 1984, Loridan [13] studied the concept of $\epsilon$-efficient solutions for vector minimization problems where the function to be optimized has its values in the $R^{n}$ space, which is a generalization of the classical problem for Pareto solution. Later in 1986, White [14] extended $\epsilon$-optimality for scalar problems to vector maximization problems, or efficiency problems, with $m$ objective functions defined on a subset of $R^{n}$. In 1993, Burke et al. [15] studied the concept of weak sharp minima for scalar optimization problem which was motivated by the application in convex and convex composite mathematical programming.

Recently, in 2016, Zhu [16] suggested the necessary optimal conditions for the weak local sharp efficient solution of a constrained multi-objective optimization problem by using the generalized Fermat formula, the Mordukhovich subdifferential for maximum functions, the fuzzy sum rule for Fréchet subdifferentials, and the sum rule for Mordukhovich subdifferentials, and also got the some sufficient optimal conditions respectively for the local and global weak sharp efficient solutions of such a multi-objective optimization problem, by applying the approximate projection method, and some appropriate convexity and affineness conditions.

Motivated by the ideas of local sharp and weak local sharp efficient solutions, we define the local sharp vector variational type inequalities and Minty local sharp vector variational type inequalities, and establish the relations between local (or Minty local) sharp vector variational type inequality and vector optimization problems involving generated by locally Lipschitzian mappings. 


\section{Preliminaries}

Throughout this paper, $\mathbb{R}^{n}$ denotes the $n$-dimensional Euclidean space with a norm $\|\cdot\|$. Let $X$ be a nonempty convex subset of $\mathbb{R}^{n}$. The distance function $d(\cdot, X): X \rightarrow \mathbb{R}$ is defined by

$$
d(x, X)=\inf _{x_{0} \in X}\left\|x-x_{0}\right\|, \quad \forall x \in X
$$

A vector valued function $\eta: X \times X \rightarrow X$ is said to be $\tau$-Lipschitz continuous if there exists a number $\tau>0$ such that

$$
\|\eta(x, y)\| \leq \tau\|x-y\|, \forall x, y \in X .
$$

Definition 1. Let $\eta: X \times X \rightarrow X$ be a function. A lower semicontinuous function $\varphi: X \rightarrow \mathbb{R}$ is said to be approximate $\eta$-convex at $x_{0} \in X$ if for any $\tau>0$, there exists $\delta>0$, such that, for all $x, y \in B\left(x_{0}, \delta\right) \cap X$,

$$
\varphi(y) \geq \varphi(x)+\left\langle x^{\star}, \eta(y, x)\right\rangle-\tau\|y-x\|, \forall x^{*} \in \partial \varphi(x) .
$$

Definition 2. Let $\eta: X \times X \rightarrow X$ be a function. A function $\varphi: X \rightarrow \mathbb{R}$ is said to be

(i) approximate $\eta$-pseudoconvex type-I at $x_{0} \in X$ if for any $\tau>0$, there exists $\delta>0$, such that, whenever $x, y \in B\left(x_{0}, \delta\right) \cap X$ and

$$
\left\langle x^{*}, \eta(y, x)\right\rangle \geq 0, \text { for some } x^{*} \in \partial \varphi(x),
$$

then

$$
\varphi(y)-\varphi(x) \geq-\tau\|y-x\|
$$

(ii) approximate $\eta$-pseudoconvex type-II (strictly approximate $\eta$-pseudoconvex type-II) at $x_{0} \in X$ if for any $\tau>0$, there exists $\delta>0$, such that, whenever $x, y \in B\left(x_{0}, \delta\right) \cap X$ and

$$
\left\langle x^{*}, \eta(y, x)\right\rangle+\tau\|y-x\| \geq 0, \text { for some } x^{*} \in \partial \varphi(x),
$$

then

$$
\varphi(y) \geq(>) \varphi(x)
$$

(iii) approximate $\eta$-quasiconvex type-I at $x_{0} \in X$ if for any $\tau>0$, there exists $\delta>0$, such that, whenever $x, y \in B\left(x_{0}, \delta\right) \cap X$ and

$$
\varphi(y) \leq \varphi(x)
$$

then

$$
\left\langle x^{*}, \eta(y, x)\right\rangle-\tau\|y-x\| \leq 0, \forall x^{*} \in \partial \varphi(x) ;
$$

(iv) approximate $\eta$-quasiconvex type-II ( strictly approximate $\eta$-quasiconvex type-II) at $x_{0} \in X$ if for any $\tau>0$, there exists $\delta>0$, such that, whenever $x, y \in B\left(x_{0}, \delta\right) \cap X$ and

$$
\varphi(y) \leq(<) \varphi(x)+\tau\|y-x\|
$$

then

$$
\left\langle x^{*}, \eta(y, x)\right\rangle \leq 0, \forall x^{*} \in \partial \varphi(x) .
$$

(VOP): A vector optimization problem (VOP) is formulated as follows:

$$
\left\{\begin{array}{l}
\operatorname{Min} f(x), \\
\text { Subject to } x \in X \subset \mathbb{R}^{n},
\end{array}\right.
$$

where, $f ; X \subset R^{n} \rightarrow R^{p}$ with $f(x)=\left(f_{1}(x), \cdots, f_{p}(x)\right)$, is a vector valued function. 
Definition 3 ([16]).

(i) A vector $x_{0} \in X$ is said to be a local sharp efficient solution of (VOP), if for any $\tau>0$ there exists a $\delta$-neighborhood of $x_{0}$, such that for all $x \in B\left(x_{0}, \delta\right) \cap X$,

$$
\max _{1 \leq i \leq p}\left\{f_{i}(x)-f_{i}\left(x_{0}\right)\right\} \geq \tau\left\|x-x_{0}\right\|
$$

(ii) A vector $x_{0} \in X$ is said to be a weak local sharp efficient solution of (VOP), if for any $\tau>0$, there exists a $\delta$-neighborhood of $x_{0}$, such that for all $x \in B\left(x_{0}, \delta\right) \cap X$,

$$
\max _{1 \leq i \leq p}\left\{f_{i}(x)-f_{i}\left(x_{0}\right)\right\} \geq \tau d(x, \bar{X}),
$$

where

$$
\bar{X}:=\left\{x \in X \mid f(x)=f\left(x_{0}\right)\right\}=X \cap f^{-1}\left(f\left(x_{0}\right)\right) .
$$

\section{Local Sharp Vector Variational Type Inequalities}

In this section, we consider local sharp and weak local sharp formulations of vector variational type inequality problems as follows:

(LSVVTI): For finding $x_{0} \in X$, there exists a $\delta$-neighborhood of $x_{0}$ and for any $\tau>0$, such that $x \in B\left(x_{0}, \delta\right) \cap X$ and

$$
\max _{1 \leq i \leq p} \max _{x_{0_{i}}^{*} \in \partial f_{i}\left(x_{0}\right)}\left\langle x_{0_{i}}^{*}, \eta\left(x, x_{0}\right)\right\rangle \geq \tau\left\|x-x_{0}\right\|, \forall x_{0_{i}}^{*} \in \varphi f_{i}\left(x_{0}\right) .
$$

(WLSVVTI): For finding $x_{0} \in X$, there exists a $\delta$-neighborhood of $x_{0}$ and for any $\tau>0$, such that $x \in B\left(x_{0}, \delta\right) \cap X$ and

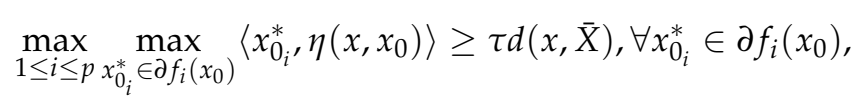

where

$$
\bar{X}=\left\{x \in X \mid f(x)=f\left(x_{0}\right)\right\}=X \cap f^{-1}\left(f\left(x_{0}\right)\right) .
$$

We note that, if $x_{0}$ is a solution of (LSVVTI), then $x_{0}$ is also a solution of (WLSVVTI).

Special Cases: Assume that, if $\eta\left(x, x_{0}\right)=x-x_{0}$. Then,

- (1) reduces to local sharp vector variational inequalities (LSVVI): for finding $x_{0} \in X$, there exists a $\delta$-neighborhood of $x_{0}$ and for any $\tau>0$, such that $x \in B\left(x_{0}, \delta\right) \cap X$ and

$$
\max _{1 \leq i \leq p} \max _{x_{0_{i}}^{*} \in \partial f_{i}\left(x_{0}\right)}\left\langle x_{0_{i^{\prime}}}^{*}, x-x_{0}\right\rangle \geq \tau\left\|x-x_{0}\right\|, \forall x_{0_{i}}^{*} \in \varphi f_{i}\left(x_{0}\right) .
$$

- In addition, (2) reduces to weak local sharp vector variational inequalities (WLSVVI) for finding $x_{0} \in X$, there exists a $\delta$-neighborhood of $x_{0}$ and for any $\tau>0$, such that $x \in B\left(x_{0}, \delta\right) \cap X$ and

$$
\max _{1 \leq i \leq p} \max _{x_{0_{i}}^{*} \in \partial f_{i}\left(x_{0}\right)}\left\langle x_{0_{i}}^{*}, x-x_{0}\right\rangle \geq \tau d(x, \bar{X}), \forall x_{0_{i}}^{*} \in \partial f_{i}\left(x_{0}\right),
$$

where

$$
\bar{X}=\left\{x \in X \mid f(x)=f\left(x_{0}\right)\right\}=X \cap f^{-1}\left(f\left(x_{0}\right)\right) .
$$


- Again, we note that if $\eta\left(x, x_{0}\right)=x-x_{0}$, then the solution of (LSVVI) is also a solution of (AVVI) (defined by [17]), but the converse need not be true:

Example: consider the function

$$
f(x)=\left(f_{1}(x), f_{2}(x)\right), x \in \mathbb{R},
$$

where $f_{1}(x)=|x|-x^{2}$ and $f_{2}(x)=-x^{2}$.

If we take $x_{0}=0$, then for any $\tau>0$, there does not exist any $\delta>0$ such that

$$
\left\langle x_{0_{i}}^{*}, x-x_{0}\right\rangle \leq \tau\left\|x-x_{0}\right\|, \forall i \in\{1,2\}, x_{0_{i}}^{*} \in \partial f_{i}\left(x_{0}\right), x \in B\left(x_{0}, \delta\right) \cap \mathbb{R},
$$

that is, $x_{0}$ is a solution of $(\mathrm{AVVI})_{1}$. When $x<0$, then for every $\delta>0$ and $\tau>0$, we do not have

$$
\max _{1 \leq i \leq p} \max _{x_{0_{i}}^{*} \in \partial f_{i}\left(x_{0}\right)}\left\langle x_{0_{i}}^{*}, x-x_{0}\right\rangle \geq \tau\left\|x-x_{0}\right\|,
$$

that is, $x_{0}$ is not a solution of (LSVVI).

Unless otherwise stated, the following condition (C) is always assumed in this section.

(C) For the bi-function $\eta: X \times X \rightarrow X$ and the mappings $f_{i}: X \rightarrow \mathbb{R}, i=1, \cdots, p$,

$$
\left\langle f_{i}(x), \eta(x, x)\right\rangle=0
$$

for all $x \in X$.

First of all, in this section, we give the relationship between the solutions of local sharp vector variational type inequalities (LSVVTI) and local sharp (or weak local sharp) efficient solutions of vector optimization problem (VOP).

Now we are at the stage of introducing and proving the main theorems:

Theorem 4. Let $\eta: X \times X \rightarrow X$ be a function and $f_{i}: X \rightarrow \mathbb{R}, i=1, \cdots, p$ be locally Lipschitz and approximate $\eta$-convex at $x_{0} \in X$, and satisfies the condition (C). If $x_{0}$ solves (LSVVTI), then it is a local sharp efficient solution of $(V O P)$.

Proof. Contrary, assume that $x_{0} \in X$ is not a local sharp efficient solution of (VOP). Then, for any $\delta_{0}>0$ and $\frac{\tau}{2}>0$, there exists $x \in B\left(x_{0}, \delta_{0}\right) \cap X$ such that

$$
\max _{1 \leq i \leq p}\left\{f_{i}(x)-f_{i}\left(x_{0}\right)\right\}<\frac{\tau}{2}\left\|x-x_{0}\right\|,
$$

it implies,

$$
f_{i}(x)-f_{i}\left(x_{0}\right)<\frac{\tau}{2}\left\|x-x_{0}\right\|
$$

Since $f_{i}$ is approximate $\eta$-convex at $x_{0} \in X$, there exists $\bar{\delta}_{i}>0$ such that for $\delta:=\min \left\{\delta_{0}, \bar{\delta}_{i}: i=\right.$ $1, \cdots, p\}$, we have

$$
f_{i}(x) \geq f_{i}\left(x_{0}\right)+\left\langle x_{0_{i}}^{*}, \eta\left(x, x_{0}\right)\right\rangle-\frac{\tau}{2}\left\|x-x_{0}\right\|, \forall x \in B\left(x_{0}, \delta\right) \cap X \text { and } x_{0_{i}}^{*} \in \partial f_{i}\left(x_{0}\right) .
$$

Hence, it follows from (5) and (6) that

$$
\frac{\tau}{2}\left\|x-x_{0}\right\|>\left\langle x_{0_{i}}^{*}, \eta\left(x, x_{0}\right)\right\rangle-\frac{\tau}{2}\left\|x-x_{0}\right\| .
$$

Therefore, we have

$$
\tau\left\|x-x_{0}\right\|>\left\langle x_{0_{i}}^{*}, \eta\left(x, x_{0}\right)\right\rangle,
$$


it implies that

$$
\max _{1 \leq i \leq p} \max _{x_{0_{i}}^{*} \in \partial f_{i}\left(x_{0}\right)}\left\langle x_{0_{i}}^{*}, \eta\left(x, x_{0}\right)\right\rangle<\tau\left\|x-x_{0}\right\|, \forall x \in B\left(x_{0}, \delta\right) \cap X \text { and } x_{0_{i}}^{*} \in \partial f_{i}\left(x_{0}\right) .
$$

This is a contradiction to the fact that $x_{0}$ solves (LSVVTI).

In following theorem, we obtain the converse result of Theorem 4 by assuming the approximate $\eta$-convexity of $-f_{i}$ instead of $f_{i}$.

Theorem 5. For each $i=1, \cdots, p$, let $\eta$ and $f_{i}$ be same as in Theorem $4,-f_{i}$ be approximate $\eta$-convex at $x_{0} \in X$, and satisfies the condition (C). Then the converse statement of Theorem 4 is true.

Proof. Suppose that $x_{0} \in X$ is not a solution of the (LSVVTI). Then, for any $\delta_{0}>0$ and $\frac{\tau}{2}>0$, there exists $x \in B\left(x_{0}, \delta_{0}\right) \cap X$ and $x_{0_{i}}^{*} \in \partial f_{i}\left(x_{0}\right)$, such that

$$
\max _{1 \leq i \leq p} \max _{x_{0_{i}}^{*} \in \partial f_{i}\left(x_{0}\right)}\left\langle x_{0_{i}}^{*}, \eta\left(x, x_{0}\right)\right\rangle<\frac{\tau}{2}\left\|x-x_{0}\right\|,
$$

it implies

$$
\left\langle x_{0_{i}}^{*}, \eta\left(x, x_{0}\right)\right\rangle<\frac{\tau}{2}\left\|x-x_{0}\right\| .
$$

Since $-f_{i}$ is approximate $\eta$-convex at $x_{0} \in X$, for any $\frac{\tau}{2}>0$, there exists $\bar{\delta}_{i}>0$, such that for $\delta:=\min \left\{\delta_{0}, \bar{\delta}_{i}: i=1, \cdots p\right\}$, we have

$$
-f_{i}(x) \geq-f_{i}\left(x_{0}\right)+\left\langle x_{0_{i}}^{*}, \eta\left(x, x_{0}\right)\right\rangle-\frac{\tau}{2}\left\|x-x_{0}\right\|, \forall x \in B\left(x_{0}, \delta\right) \cap X \text { and } x_{0_{i}}^{*} \in-\partial f_{i}\left(x_{0}\right),
$$

we can write it as

$$
\left\langle x_{0_{i}}^{*}, \eta\left(x, x_{0}\right)\right\rangle \geq f_{i}(x)-f_{i}\left(x_{0}\right)-\frac{\tau}{2}\left\|x-x_{0}\right\| .
$$

From (7) and (8), we have

$$
\frac{\tau}{2}\left\|x-x_{0}\right\|>f_{i}(x)-f_{i}\left(x_{0}\right)-\frac{\tau}{2}\left\|x-x_{0}\right\|, \forall x \in B\left(x_{0}, \delta\right) \cap X .
$$

Hence, we have

$$
f_{i}(x)-f_{i}\left(x_{0}\right)<\tau\left\|x-x_{0}\right\|,
$$

this implies that

$$
\max _{1 \leq i \leq p}\left\{f_{i}(x)-f_{i}\left(x_{0}\right)\right\}<\tau\left\|x-x_{0}\right\|, \forall x \in B\left(x_{0}, \delta\right) \cap X,
$$

which is a contradiction to the fact that $x_{0}$ is a local sharp efficient solution of (VOP).

In next theorem, the same result of Theorem 4 is obtained by substituting the strictly approximate $\eta$-quasiconvex type-II condition instead of approximate $\eta$-convexity condition on $f_{i}$.

Theorem 6. Let $\eta$ and $f_{i}$ be the same as in Theorem $4, f_{i}: X \rightarrow \mathbb{R}$ be a strictly approximate $\eta$-quasiconvex type-II at $x_{0} \in X$, for each $i=1, \cdots, p$, and satisfies the condition (C). If $x_{0}$ solves (LSVVTI), then it is a local sharp efficient solution of (VOP).

Proof. Assume that $x_{0} \in X$ is not a local sharp efficient solution of (VOP). Then, for any $\delta_{0}>0$ and $\tau>0$, there exists $x \in B\left(x_{0}, \delta_{0}\right) \cap X$, such that

$$
\max _{1 \leq i \leq p}\left\{f_{i}(x)-f_{i}\left(x_{0}\right)\right\}<\tau\left\|x-x_{0}\right\|,
$$


it implies,

$$
f_{i}(x)-f_{i}\left(x_{0}\right)<\tau\left\|x-x_{0}\right\| .
$$

Since $f_{i}$ is a strictly approximate $\eta$-quasiconvex type-II at $x_{0} \in X$, for any $\tau>0$, there exists $\bar{\delta}_{i}>0$, such that by setting $\delta=\min \left\{\delta_{0}, \bar{\delta}_{i}: i=1, \cdots, p\right\}$, we have

$$
\left\langle x_{0_{i}}^{*}, \eta\left(x, x_{0}\right)\right\rangle \leq 0<\tau\left\|x-x_{0}\right\|, \forall x \in B\left(x_{0}, \delta\right) \cap X \text { and } x_{0_{i}}^{*} \in \partial f_{i}\left(x_{0}\right),
$$

implies that

$$
\max _{1 \leq i \leq p} \max _{x_{0_{i}}^{*} \in \partial f_{i}\left(x_{0}\right)}\left\langle x_{0_{i}}^{*}, \eta\left(x, x_{0}\right)\right\rangle<\tau\left\|x-x_{0}\right\|, \forall x \in B\left(x_{0}, \delta\right) \cap X \text { and } x_{0_{i}}^{*} \in \partial f_{i}\left(x_{0}\right) .
$$

This means that $x_{0}$ is not a solution of (LSVVTI).

In the following theorem, we can get the the generalization of Theorem 5 by assuming the strictly approximate $\eta$-pseudoconvex type-II condition on $-f_{i}$.

Theorem 7. For each $i=1, \cdots, p$, let $\eta$ and $f_{i}$ be same as in Theorem $4,-f_{i}$ be a strictly approximate $\eta$-pseudoconvex type-II at $x_{0} \in X$, and satisfies the condition (C). If $x_{0}$ is a weak local sharp efficient solution of (VOP), then it is also a solution of (LSVVTI).

Proof. Suppose that $x_{0} \in X$ is not a solution of (LSVVTI). Then, for any $\delta_{0}>0$ and $\tau>0$, there exists $x \in B\left(x_{0}, \delta_{0}\right) \cap X$ and $x_{0_{i}}^{*} \in \partial f_{i}\left(x_{0}\right)$, such that

$$
\max _{1 \leq i \leq p} \max _{x_{0_{i}}^{*} \in \partial f_{i}\left(x_{0}\right)}\left\langle x_{0_{i}}^{*}, \eta\left(x, x_{0}\right)\right\rangle<\tau\left\|x-x_{0}\right\| .
$$

Hence, we have,

$$
\left\langle x_{0_{i}}^{*}, \eta\left(x, x_{0}\right)\right\rangle<\tau\left\|x-x_{0}\right\|,
$$

and we can rewrite as

$$
\left\langle-x_{0_{i}}^{*} \eta \eta\left(x, x_{0}\right)\right\rangle+\tau\left\|x-x_{0}\right\|>0 .
$$

Since $-f_{i}$ is a strictly approximate $\eta$-pseudoconvex type-II at $x_{0} \in X$, for any $\tau>0$, there exists $\bar{\delta}_{i}>0$ such that, for $\delta:=\min \left\{\delta_{0}, \bar{\delta}_{i}: i=1, \cdots, p\right\}$, we have

$$
-f_{i}(x)>-f_{i}\left(x_{0}\right), \forall x \in B\left(x_{0}, \delta\right) \cap X .
$$

Therefore, we have

$$
f_{i}(x)-f_{i}\left(x_{0}\right)<0 \leq \tau d(x, \bar{X}),
$$

this implies that

$$
\max _{1 \leq i \leq p}\left\{f_{i}(x)-f_{i}\left(x_{0}\right)\right\}<\tau d(x, \bar{X}), \forall x \in B\left(x_{0}, \delta\right) \cap X .
$$

Therefore, we show that $x_{0}$ is a local weak sharp efficient solution of (VOP). This completes the proof.

\section{Minty Local Sharp Vector Variational Type Inequalities}

In this section, we present relationship between the solutions of Minty local sharp vector variational type inequalities (MLSVVTI) and local sharp (or weak local sharp) efficient solutions of vector optimization problem (VOP).

Now, we consider Minty local sharp and Minty weak local sharp formulations of vector variational type inequality problems as follows: 
(MLSVVTI): Finding $x_{0} \in X$, there exists a $\delta$-neighborhood of $x_{0}$ and any $\tau>0$, such that $x \in$ $B\left(x_{0}, \delta\right) \cap X$ and

$$
\max _{1 \leq i \leq p} \max _{i}^{* * \partial f_{i}(x)}\left\langle x_{i}^{*}, \eta\left(x, x_{0}\right)\right\rangle \geq \tau\left\|x-x_{0}\right\|, \quad \forall x_{i}^{*} \in \partial f_{i}(x) .
$$

(MWLSVVTI): For finding $x_{0} \in X$, there exists a $\delta$-neighborhood of $x_{0}$ and any $\tau>0$, such that $x \in B\left(x_{0}, \delta\right) \cap X$ and

$$
\max _{1 \leq i \leq p} \max _{x_{i}^{*} \in \partial f_{i}(x)}\left\langle x_{i}^{*}, \eta\left(x, x_{0}\right)\right\rangle \geq \tau d(x, \bar{X}), \quad \forall x_{i}^{*} \in \partial f_{i}(x),
$$

where $\bar{X}=\left\{x \in X \mid f(x)=f\left(x_{0}\right)\right\}=X \cap f^{-1}\left(f\left(x_{0}\right)\right)$.

Theorem 8. For each $i=1, \cdots, p$, let $\eta$ and $f_{i}$ be same as in Theorem $4,-f_{i}$ be approximate $\eta$-convex at $x_{0} \in X$, and satisfies the condition (C). If $x_{0}$ solves (MLSVVTI), then $x_{0}$ is a local sharp efficient solution of $(V O P)$.

Proof. Suppose that $x_{0} \in X$ is not a local sharp efficient solution of (VOP). Then, for any $\delta_{0}>0$ and $\frac{\tau}{2}>0$, there exists $x \in B\left(x_{0}, \delta_{0}\right) \cap X$, such that

$$
\max _{1 \leq i \leq p}\left\{f_{i}(x)-f_{i}\left(x_{0}\right)\right\}<\frac{\tau}{2}\left\|x-x_{0}\right\|,
$$

it implies,

$$
f_{i}(x)-f_{i}\left(x_{0}\right)<\frac{\tau}{2}\left\|x-x_{0}\right\|
$$

Since $-f_{i}$ is approximate $\eta$-convex at $x_{0} \in X$, for any $\frac{\tau}{2}>0$, there exists $\bar{\delta}_{i}>0$, such that, for $\delta:=\min \left\{\delta_{0}, \bar{\delta}_{i}: i=1, \cdots, p\right\}$, we have

$$
-f_{i}\left(x_{0}\right) \geq-f_{i}(x)+\left\langle-x_{i}^{*}, \eta\left(x_{0}, x\right)\right\rangle-\frac{\tau}{2}\left\|x_{0}-x\right\|, \forall x \in B\left(x_{0}, \delta\right) \cap X \text { and }-x_{i}^{*} \in-\partial f_{i}(x) .
$$

It follows from (14) and (15), we have

$$
\frac{\tau}{2}\left\|x-x_{0}\right\|>\left\langle-x_{i}^{*}, \eta\left(x_{0}, x\right)\right\rangle-\frac{\tau}{2}\left\|x_{0}-x\right\|, \forall x \in B\left(x_{0}, \delta\right) \cap X \text { and }-x_{i}^{*} \in-\partial f_{i}(x),
$$

that is,

$$
\tau\left\|x-x_{0}\right\|>\left\langle-x_{i}^{*}, \eta\left(x_{0}, x\right)\right\rangle
$$

implies that

$$
\max _{1 \leq i \leq p} \max _{x_{i}^{*} \in \partial f_{i}(x)}\left\langle x_{i}^{*}, \eta\left(x, x_{0}\right)\right\rangle<\tau\left\|x-x_{0}\right\|, \forall x \in B\left(x_{0}, \delta\right) \cap X \text { and } x_{i}^{*} \in \partial f_{i}(x),
$$

which is a contradiction to the fact that $x_{0}$ solves (MLSVVTI). This completes the proof.

In following theorem, we can get the converse result of Theorem 8 by assuming the approximate $\eta$-convexity of $f_{i}$ instead of $-f_{i}$.

Theorem 9. For each $i=1, \cdots, p$, let $\eta$ and $f_{i}$ be same as in Theorem $8, f_{i}: X \rightarrow \mathbb{R}$ be approximate $\eta$-convex at $x_{0} \in X$, and satisfies the condition (C). If $x_{0}$ is a local sharp efficient solution of $(V O P)$, then $x_{0}$ solves (MLSVVTI). 
Proof. Suppose that $x_{0} \in X$ is not a solution of the (MLSVVTI). Then, for any $\delta_{0}>0$ and $\frac{\tau}{2}>0$, there exists $x \in B\left(x_{0}, \delta_{0}\right) \cap X$ and $x_{i}^{*} \in \partial f_{i}(x)$, such that

$$
\max _{1 \leq i \leq p} \max _{x_{i}^{*} \in \partial f_{i}(x)}\left\langle x_{i}^{*}, \eta\left(x, x_{0}\right)\right\rangle<\frac{\tau}{2}\left\|x-x_{0}\right\|,
$$

it implies,

$$
\left\langle x_{i}^{*}, \eta\left(x, x_{0}\right)\right\rangle<\frac{\tau}{2}\left\|x-x_{0}\right\| .
$$

Since $f_{i}$ is approximate $\eta$-convex at $x_{0} \in X$, for any $\frac{\tau}{2}>0$, there exists $\bar{\delta}_{i}>0$, such that, for $\delta:=\min \left\{\delta_{0}, \bar{\delta}_{i}: i=1, \cdots, p\right\}$, we have

$$
f_{i}\left(x_{0}\right) \geq f_{i}(x)+\left\langle x_{i}^{*}, \eta\left(x_{0}, x\right)\right\rangle-\frac{\tau}{2}\left\|x_{0}-x\right\|, \forall x \in B\left(x_{0}, \delta\right) \cap X \text { and } x_{i}^{*} \in \partial f_{i}(x),
$$

we can rewrite as

$$
\left\langle x_{i}^{*}, \eta\left(x, x_{0}\right)\right\rangle \geq f_{i}(x)-f_{i}\left(x_{0}\right)-\frac{\tau}{2}\left\|x-x_{0}\right\| .
$$

Combining (16) and (17), we have

$$
\frac{\tau}{2}\left\|x-x_{0}\right\|>f_{i}(x)-f_{i}\left(x_{0}\right)-\frac{\tau}{2}\left\|x-x_{0}\right\|, \forall x \in B\left(x_{0}, \delta\right) \cap X .
$$

Hence, we have

$$
f_{i}(x)-f_{i}\left(x_{0}\right)<\tau\left\|x-x_{0}\right\|,
$$

implies that

$$
\max _{1 \leq i \leq p}\left\{f_{i}(x)-f_{i}\left(x_{0}\right)\right\}<\tau\left\|x-x_{0}\right\|, \forall x \in B\left(x_{0}, \delta\right) \cap X .
$$

This is a contradiction to the fact that $x_{0}$ is a local sharp efficient solution of (VOP).

In following theorem, we can get same result of Theorem 8 by assuming the strictly approximate $\eta$-quasiconvex type-II condition insted of approximate $\eta$-convexity on $-f_{i}$.

Theorem 10. For each $i=1, \cdots, p$, let $\eta$ and $f_{i}$ be same as in Theorem $8,-f_{i}$ be a strictly approximate $\eta$-quasiconvex type-II at $x_{0} \in X$, and satisfies the condition (C). If $x_{0}$ solves (MLSVVTI), then $x_{0}$ is a local sharp efficient solution of $(V O P)$.

Proof. Assume that $x_{0} \in X$ is not a local sharp efficient solution of (VOP). Then, for any $\delta_{0}>0$ and $\tau>0$, there exists $x \in B\left(x_{0}, \delta_{0}\right) \cap X$, such that

$$
\max _{1 \leq i \leq p}\left\{f_{i}(x)-f_{i}\left(x_{0}\right)\right\}<\tau\left\|x-x_{0}\right\|,
$$

it implies,

$$
f_{i}(x)-f_{i}\left(x_{0}\right)<\tau\left\|x-x_{0}\right\|
$$

Hence, we can rewrite as

$$
-f_{i}\left(x_{0}\right)-\left(-f_{i}(x)\right)<\tau\left\|x_{0}-x\right\| .
$$

Since $-f_{i}$ is a strictly approximate $\eta$-quasiconvex type-II at $x_{0} \in X$, for any $\tau>0$, there exists $\bar{\delta}_{i}>0$ such that, for $\delta:=\min \left\{\delta_{0}, \bar{\delta}_{i}: i=1, \cdots, p\right\}$, we have

$$
\left\langle-x_{i}^{*}, \eta\left(x_{0}, x\right)\right\rangle \leq 0, \forall x \in B\left(x_{0}, \delta\right) \cap X \text { and }-x_{i}^{*} \in-\partial f_{i}(x) .
$$

That is,

$$
\left\langle x_{i}^{*}, \eta\left(x, x_{0}\right)\right\rangle \leq 0<\tau\left\|x-x_{0}\right\|
$$


implies that

$$
\max _{1 \leq i \leq p} \max _{x_{i}^{*} \in \partial f_{i}(x)}\left\langle x_{i}^{*}, \eta\left(x, x_{0}\right)\right\rangle<\tau\left\|x-x_{0}\right\|, \forall x \in B\left(x_{0}, \delta\right) \cap X \text { and } x_{i}^{*} \in \partial f_{i}(x),
$$

which is a contradiction to the fact that $x_{0}$ solves (MLSVVTI).

The following theorem is an improvement of the Theorem 9 for the weak local sharp efficient solution of (VOP).

Theorem 11. For each $i=1, \cdots, p$, let $\eta$ and $f_{i}$ be same as in Theorem $8, f_{i}: X \rightarrow \mathbb{R}$ be a strictly approximate $\eta$-pseudoconvex type-II at $x_{0} \in X$, and satisfies the condition (C). If $x_{0}$ is a weak local sharp efficient solution of (VOP), then $x_{0}$ solves (MLSVVTI).

Proof. On the contrary, assume that $x_{0} \in X$ is not a solution of (MLSVVTI). Then, for any $\delta_{0}>0$ and $\tau>0$, there exists $x \in B\left(x_{0}, \delta_{0}\right) \cap X$ and $x_{i}^{*} \in \partial f_{i}(x)$, such that

$$
\max _{1 \leq i \leq p} \max _{x_{i}^{*} \in \partial f_{i}(x)}\left\langle x_{i}^{*}, \eta\left(x, x_{0}\right)\right\rangle<\tau\left\|x-x_{0}\right\| .
$$

Hence, we obtain

$$
\left\langle x_{i}^{*}, \eta\left(x, x_{0}\right)\right\rangle<\tau\left\|x-x_{0}\right\|
$$

it implies,

$$
\left\langle x_{i}^{*}, \eta\left(x_{0}, x\right)\right\rangle+\tau\left\|x_{0}-x\right\|>0 .
$$

Since $f_{i}$ is a strictly approximate $\eta$-pseudoconvex type-II at $x_{0} \in X$, for any $\tau>0$, there exists $\bar{\delta}_{i}>0$ such that, for $\delta:=\min \left\{\delta_{0}, \bar{\delta}_{i}: i=1, \cdots, p\right\}$, we have

$$
f_{i}\left(x_{0}\right)>f_{i}(x), \forall x \in B\left(x_{0}, \delta\right) \cap X .
$$

This implies that

$$
f_{i}(x)-f_{i}\left(x_{0}\right)<0 \leq \tau d(x, \bar{X})
$$

hence, we have

$$
\max _{1 \leq i \leq p}\left\{f_{i}(x)-f_{i}\left(x_{0}\right)\right\}<\tau d(x, \bar{X}), \forall x \in B\left(x_{0}, \delta\right) \cap X
$$

This is a contradiction to the fact that $x_{0}$ is a weak local sharp efficient solution of (VOP).

\section{Conclusions}

In this paper, we formulate local (Minty local) sharp vector variational type inequality problems and establish the relationship between local (Minty local) sharp vector variational type inequality and vector optimization problems involving locally Lipschitzian functions; that is, in Theorems 4-7, we give the necessary or sufficient conditions between the local sharp vector variational type inequality (LSVVTI) and vector optimization problems (VOP), and in Theorems 8-11, we give the necessary or sufficient conditions between the Minty local sharp vector variational type inequality (MLSVVTI) and vector optimization problems (VOP), by using the approximate $\eta$-convexity, strictly approximate $\eta$-quasiconvex type-II condition, and strictly approximate $\eta$-pseudoconvex type-II condition at $x_{0} \in X$,

The results of our research in this paper are generalized, extended, and improved studies of concepts of $\epsilon$-efficient solutions for vector minimization problems [13], $\epsilon$-optimality for scalar problems to vector maximization problems, or efficiency problems [14], weak sharp minima for scalar optimization problem [15], weak local sharp efficient solution of a constrained multi-objective optimization, and the local and global weak sharp efficient solutions of such a multi-objective optimization problem [16]. 
Author Contributions: Conceptualization, J.K.K.; investigation, S. All the authors have contributed equally to this paper. All authors have read and agreed to the published version of the manuscript.

Funding: This research was funded by Ministry of Education of the republic of Korea, grant number 2018R1D1A1B07045427.

Acknowledgments: All the authors wish to express their warm thanks to the referees.

Conflicts of Interest: The authors declare no conflict of interest.

\section{References}

1. Anh, P.N.; Thach H.T.C.; Kim, J.K. Proximal-like subgradient methods for solving multi-valued variational inequalities. Nonlinear Funct. Anal. Appl. 2020, 25, 437-451.

2. Ansari, Q.H.; Ahmad, R.; Salahuddin. A perturbed Ishikawa iterative algorithm for general mixed multivalued mildly nonlinear variational inequalities. Adv. Nonlinear Var. Inequal. 2020, 3, 53-64.

3. Fan, K. A generalization of Tychonoff's fixed-point theprem. Math. Ann. 1961, 142, 305-310. [CrossRef]

4. Giannessi, F. Theorems of alternative, quadratic programs and complementarity problems. In Variational Inequalities and Complementarity Problems; Cottle, R.W., Giannessi, F., Lions, J.-L., Eds.; Wiley: New York, NY, USA, 1980; pp. 151-186.

5. Huang, H. Strict vector variational inequalities and strict Pareto efficiency in nonconvex vector optimization. Positivity 2015, 19, 95-109. [CrossRef]

6. Kim, J.K.; Salahuddin. The existence of deterministic random generalized vector equilibrium problems. Nonlinear Funct. Anal. Appl. 2015, 20, 453-464.

7. Kim, J.K.; Salahuddin. Extragradient methods for generalized mixed equilibrium problems and fixed point problems in Hilbert spaces. Nonlinear Funct. Anal. Appl. 2017, 22, 693-709.

8. Lee, B.S.; Khan, M.F.; Salahuddin. Generalized vector variational-type inequalities. Comput. Math. Appl. 2008, 55, 1164-1169. [CrossRef]

9. Lee, B.S.; Salahuddin. Minty lemma for inverted vector variational inequalities. Optimization 2017, 66, 351-359. [CrossRef]

10. Liu, Y.; Cui, Y. A modified gradient projection algorithm for variational inequalities and relatively nonexpansive mappings in Banach spaces. Nonlinear Funct. Anal. Appl. 2017, 22, 433-448.

11. Salahuddin. Convergence analysis for hierarchical optimizations. Nonlinear Anal. Forum 2015, 20, $229-239$.

12. Salahuddin. A coordinate wise variational method with tolerance functions. J. Appl. Nonlinear Dyna 2020, 9, 541-549. [CrossRef]

13. Loridan, P. $\epsilon$-solutions in vector minimization problems. J. Optim. Theory Appl. 1984, 43, 265-276. [CrossRef]

14. White, D.J. Epsilon efficiency. J. Optim. Theory Appl. 1986, 49, 319-337. [CrossRef]

15. Burke, J.V.; Ferris, M.C. Weak sharp minima in mathematical programming. SIAM J. Control Optim. 1993, 31, 1340-1359. [CrossRef]

16. Zhu, S.K. Weak sharp efficiency in multiobjective optimization. Optim. Lett. 2016, 10, 1287-1301. [CrossRef]

17. Mishra, S.K.; Laha, V. On approximately star-shaped functions and approximate vector variational inequalities. J. Optim. Theory Appl. 2013, 156, 278-293. [CrossRef]

Publisher's Note: MDPI stays neutral with regard to jurisdictional claims in published maps and institutional affiliations.

(C) 2020 by the authors. Licensee MDPI, Basel, Switzerland. This article is an open access article distributed under the terms and conditions of the Creative Commons Attribution (CC BY) license (http://creativecommons.org/licenses/by/4.0/). 